\title{
Selective digestive tract decontamination: A tough pill to swallow
}

\author{
Kevin B Laupland MD MSc FRCPC ${ }^{1}$, David N Fisman MD MPH FRCPC ${ }^{2}$
}

$\mathrm{N}$ osocomial infections are an important cause of death and excess length of stay in individuals hospitalized in the intensive care unit (ICU) (1). Ventilator-acquired pneumonia is common in this setting, occurring at an estimated incidence of 15 cases per 1000 ventilator-days (2). Notwithstanding the adoption of care guidelines that advocate best practices, such as the use of sterile water for enteral feeds, elevation of the head of the bed and optimal removal of ventilator circuit condensates, ventilator-acquired pneumonia is a frustratingly common occurrence (3). The reasons for this high burden of infectious disease in the intensive care unit extend beyond the obvious mechanical disruption of host defenses by invasive tubes and lines, and likely include the decline in immune function associated with critical illness.

A recent large, multicentred, randomized trial (4) demonstrated that the use of selective oropharyngeal decontamination (SOD) and selective digestive tract decontamination (SDD) is associated with improved mortality in critically ill adults. This study, reported by de Smet et al (4), was conducted in 13 ICUs in the Netherlands, using a 'cluster-randomized' crossover design. In other words, ICUs, rather than individual patients, were randomized to give all patients admitted over six-month periods either SDD $(n=2045)$, SOD $(n=1904)$ or standard care $(n=1990)$. Furthermore, the trial included a 'crossover' component, in which the intervention (SOD, SDD or standard care) was varied over time. This allowed the investigators to compare the effect of each intervention across ICUs, and to evaluate the effects of changing interventions within ICUs over time, which in turn helped control for differences in the 'ecology' of ICUs (eg, different levels of expertise vis-à-vis infection control, different case mix and different baseline rates of infection with drug-resistant pathogens).

Study subjects were patients with expected duration of mechanical ventilation of at least $48 \mathrm{~h}$ and/or ICU stay of more than three days. Overall, $89.2 \%$ of eligible patients were enrolled, with a slightly higher rate of inclusion of patients with standard therapy. Patients allocated to SOD had topical oropharyngeal tobramycin, colistin and amphotericin B applied throughout their ICU stay. The SDD regimen included the SOD regimen plus the addition of intravenous cefotaxime for the initial four days. The primary outcome measure of 28-day mortality was not statistically different among treatments, with rates of $27.5 \%, 26.6 \%$ and $26.9 \%$ observed with standard care, SOD and SDD, respectively. However, patients treated with SDD and SOD had greater severity of illness, as measured by baseline Acute Physiology and Chronic Health Evaluation II scores, and were more likely to be medical patients and to receive mechanical ventilation than patients treated with standard therapy. After controlling for these and a number of other potentially confounding variables using logistic regression analysis, the use of SDD and SOD was associated with statistically significant reductions in the odds of death (SOD OR 0.86, 95\% CI 0.74 to 0.99 ; SDD OR $0.83,95 \% \mathrm{CI} 0.72$ to 0.97 ). In other terms, SOD and SDD use was associated with absolute risk reductions of $2.9 \%$ and $3.5 \%$, corresponding to 34 and 29 patients treated to prevent one death, respectively.

This study is important because it concurrently compared SOD and SDD, and added further evidence to the growing body of published literature $(5,6)$ supporting mortality benefits with decontamination therapies. In 2004, Liberati et al (7), on behalf of the Cochrane collaboration, conducted a meta-analysis of clinical trials comparing prophylactic antibiotic regimens in adults admitted to the ICU. They reviewed 17 trials (4075 patients) evaluating topical plus systemic prophylaxis (SDD) compared with no prophylaxis, and found significant overall reductions in mortality (OR 0.75; 95\% CI 0.65 to 0.87 ) corresponding to a number needed-to-treat of 21 patients to prevent one death. Although SOD reduced the risk for infection in 13 included trials (1597 patients), it alone did not significantly reduce mortality (OR 0.96; 95\% CI 0.78 to 1.20 ) compared with no decontamination or placebo. Since then, there have been other published trials that further support the benefits of decontamination therapy (8-10).

Do these data justify the routine use of SOD and/or SDD in critically ill adults at risk for prolonged ICU stays? The rationale underlying decontamination therapies seems plausible. Most ICU-acquired infections, particularly those acquired relatively early in the ICU stay, are 'endogenous' that is, they are caused by the patient's own oropharyngeal and/or intestinal flora. While important, interventions such as enhanced hand hygiene in health care workers may be expected to have a limited effect on reducing the risk of such infections (11). The goal of SOD is to prevent infection by suppression or reduction of this colonization by yeasts and aerobic Gram-negative bacteria by application of nonabsorbable topical antimicrobials. The rationale for the addition of systemic antibiotherapy in SDD is to also preemptively treat incubating infections; in a sense, this systemic therapy can be

\footnotetext{
${ }^{1}$ Departments of Medicine, Critical Care Medicine, Pathology and Laboratory Medicine, Centre for Antimicrobial Resistance, University of Calgary, Calgary Laboratory Services, Calgary Health Region, Calgary, Alberta; ${ }^{2}$ The Research Institute of the Hospital for Sick Children, University of Toronto, Ontario Agency for Health Protection and Promotion, Toronto, Ontario

Correspondence: Dr Kevin B Laupland, Room 719, North Tower, Foothills Medical Centre, 1403 - 29th Street Northwest, Calgary,

Alberta T2N 2T9. Telephone 403-944-5808, fax 403-944-3199, e-mail kevin.laupland@calgaryhealthregion.ca

Received and accepted for publication February 26, 2009
} 
regarded as analogous to 'postexposure antimicrobial prophylaxis' applied to individuals (eg, those who have sustained animal bites) who have experienced exposures highly likely to result in severe infection.

There are also a number of clinical considerations that argue in favour of use of decontamination therapies. Our ability to detect and adequately treat serious infections in critically ill patients in a timely manner is limited, and a preventive approach has intrinsic appeal. Kumar et al (12) conducted a retrospective cohort study of 2731 patients with septic shock who were admitted to 14 ICUs in Canada and the United States, and found that the time to initiation of effective antimicrobial therapy after onset of hypotension was the most important determinant of outcome. They found that for every hour of delay in administration of effective antimicrobial therapy, the odds for death increased by 1.12 (95\% CI 1.10 to 1.14). Invasive fungal infections are occult to diagnosis by blood cultures in approximately one-third of cases in critically ill adults and are not uncommonly first identified at autopsy. Even when blood culture-positive cases are studied, failure to initiate adequate empirical therapy is commonplace and associated with an adverse outcome (13). By either preemptively treating or preventing infections, SOD and SDD may compensate at least in part for our difficulty as clinicians to consistently detect and initiate early appropriate therapy in septic patients. Second, SOD and SDD significantly reduce the incidence of ICU-acquired infections. Even if clinicians are unconvinced by evidence pointing to reduced mortality, they clearly reduce the occurrence and morbidity associated with complicating infections, most importantly, bloodstream infection and pneumonia. In the de Smet et al (4) study, the incidence of ICU-acquired bacteremia or fungemia was significantly reduced with SDD (OR 0.44; 95\% CI 0.34 to 0.57 ) and SOD (OR 0.68; 95\% CI 0.53 to 0.86 ) compared with standard care. These results confirm the results of meta-analyses of previous trials that also show an approximate halving of the risk for pneumonia associated with decontamination therapies (7). The 'attribution of mortality' in the case of ICU-acquired infections may be difficult to demonstrate because individuals at highest risk of acquiring nosocomial infections may also be at highest risk of death from other causes, due to underlying severity of illness. However, there is good reason to hope to prevent infections acquired in the ICU because these infections prolong ICU and hospital stays, result in patient discomfort through increased investigation and procedural interventions, and increase costs of care.

So why are SOD and SDD not used routinely in adult ICUs in Canada and in most jurisdictions worldwide? Guidelines have consistently either neglected or deferred recommendations on the use of decontamination therapies (14-16). While there are a number of considerations, the most important surrounds the risk for promotion of antimicrobial resistance. The concept of exposing vast numbers of critically ill patients to broad-spectrum multiple drug cocktails runs counter to existing theoretical models (and dogma) related to the genesis and promotion of antimicrobial resistance in pathogens acquired in the health care setting. Proponents of SOD/SDD might argue that the provision of SOD/SDD would be a 'zero-sum game' with respect to antimicrobial exposure in the ICU - SOD/SDD recipients would certainly receive less antimicrobials for the treatment of downstream infections; furthermore, by reducing gut colonization of patients with Gram-negative rods and yeasts with high pathogenic potential, there are indirect 'herd' effects that prevent transmission of pathogens to other patients via breaks in hand hygiene or other infection control techniques.

Indeed, the available evidence does not support the contention that utilization of SOD and SDD would necessarily result in surging infection rates with antimicrobial-resistant pathogens, although this issue deserves further study because relatively few trials of SOD/SDD have systematically addressed this question. de Jonge et al (5) conducted a prospective open-label clinical trial, in which 934 critically ill adult patients were randomly assigned to admission to either a medical/surgical ICU using routine SDD or a similar ICU in the same hospital that did not use SDD. Enrollment criteria and the SDD regimen were similar to the de Smet et al study (4). Surveillance cultures from the sputum, throat, rectum, axilla and wounds were obtained at ICU admission and discharge, weekly during the ICU stay and for the first week postdischarge. The in-hospital mortality was significantly lower for SDD compared with control patients (24\% versus $31 \% ; \mathrm{P}=0.02$ ). Colonization with resistant (to ceftazidime, ciprofloxacin, imipenem, polymyxin E or tobramycin) Gramnegative bacteria was reduced in SDD patients compared with controls (16\% versus 26\%; $\mathrm{P}=0.001)$. Colonization with vancomycin-resistant enterococcus occurred in $1 \%$ of patients in each group and none with methicillin-resistant Staphylococcus aureus (MRSA). Similar results were observed by de Smet et al (4) in their cluster randomized trial. Monthly point prevalence surveys for resistant organisms were obtained. The proportion of rectal swabs with resistant Gram-negative organisms was lower for SDD compared with SOD and standard care, and was lower for SOD and SDD compared with standard care for respiratory specimens. Colonization with vancomycin-resistant enterococcus was rare in all groups (less than 1\%), and no patients had MRSA. The absence of MRSA detection in either the de Jonge (5) or de Smet et al (4) studies has been flagged as an important concern by those who regard SOD/SDD as an entity that requires additional study before widespread adoption (17). The experience in these trials, vis-à-vis MRSA, represents a distinctly different experience than in many other jurisdictions in which MRSA is an increasing and major cause of ICU. acquired infections.

However, as van Saene et al (11) argued in a narrative review several years ago, ongoing surveillance efforts to monitor the success of SOD/SDD and identify the emergence of antimicrobialresistant threats is an intrinsic component of any SOD/SDD program, and in this sense, a well-designed program contains an intrinsic degree of protection against antibiotic-resistant organism emergence. Furthermore, these SOD/SDD advocates argue that the targeted nature of SOD/SDD regimens is likely to preserve patient flora with low pathogenic potential, which provides additional protection against colonization and/or overgrowth of drug-resistant organisms.

So what should we do? On one hand, decontamination therapy significantly reduces the occurrence and morbidity associated with ICU-acquired infections, and likely reduces mortality among adults admitted to ICU with expected prolonged stays. On the other hand, the prophylactic application of large doses of broad-spectrum antimicrobial agents carries 
the theoretical potential for increasing the prevalence of infection with antimicrobial-resistant organisms, and perhaps more influential in practice, is at odds with our traditional fundamentals of antimicrobial stewardship. From an objective, strictly evidence-based perspective, one is left to conclude that SOD/SDD should be recommended because there is level 1 clinical trial evidence supporting its use but only expert opinion against it (11). Stated differently, at this point, there is a high degree of certainty that SOD/SDD will benefit the individual patient, while carrying only the potential and theoretical future risk of harm to other patients, the community

\section{REFERENCES}

1. Richards MJ, Edwards JR, Culver DH, Gaynes RP. Nosocomial infections in combined medical-surgical intensive care units in the United States. Infect Control Hosp Epidemiol 2000;21:510-5.

2. Cook DJ, Walter SD, Cook RJ, et al. Incidence of and risk factors for ventilator-associated pneumonia in critically ill patients. Ann Intern Med 1998;129:433-40.

3. Kollef M. SMART approaches for reducing nosocomial infections in the ICU. Chest 2008;134:447-56.

4. de Smet AM, Kluytmans JA, Cooper BS, et al. Decontamination of the digestive tract and oropharynx in ICU patients. N Engl J Med 2009;360:20-31.

5. de Jonge E, Schultz MJ, Spanjaard L, et al. Effects of selective decontamination of digestive tract on mortality and acquisition of resistant bacteria in intensive care: A randomised controlled trial. Lancet 2003;362:1011-6.

6. Krueger WA, Lenhart FP, Neeser G, et al. Influence of combined intravenous and topical antibiotic prophylaxis on the incidence of infections, organ dysfunctions, and mortality in critically ill surgical patients: A prospective, stratified, randomized, double-blind, placebo-controlled clinical trial. Am J Respir Crit Care Med 2002;166:1029-37.

7. Liberati A, D’Amico R, Pifferi, Torri V, Brazzi L. Antibiotic prophylaxis to reduce respiratory tract infections and mortality in adults receiving intensive care. Cochrane Database Syst Rev 2004(1):CD000022.

8. de La Cal MA, Cerda E, Garcia-Hierro P, et al. Survival benefit in critically ill burned patients receiving selective decontamination of the digestive tract: A randomized, placebo-controlled, double-blind trial. Ann Surg 2005;241:424-30.

9. Stoutenbeek CP, van Saene HK, Little RA, Whitehead A.

The effect of selective decontamination of the digestive tract on and the patient herself/himself; this makes the ethics of withholding SOD/SDD questionable based on the best available evidence. SOD/SDD represents both a challenge and an opportunity for Canadian infectious diseases clinicians, microbiologists and intensivists. Undoubtedly, SOD/SDD will remain controversial for some time, but this concept must be brought to the forefront for vigorous debate. The onus is now on the infectious diseases and medical microbiology community to determine the long-term impact of decontamination therapies on the emergence of antimicrobial resistance.

mortality in multiple trauma patients: A multicenter randomized controlled trial. Intensive Care Med 2007;33:261-70.

10. Camus C, Bellissant E, Sebille V, et al. Prevention of acquired infections in intubated patients with the combination of two decontamination regimens. Crit Care Med 2005;33:307-14.

11. van Saene HK, Petros AJ, Ramsay G, Baxby D. All great truths are iconoclastic: Selective decontamination of the digestive tract moves from heresy to level 1 truth. Intensive Care Med 2003;29:677-90.

12. Kumar A, Roberts D, Wood KE, et al. Duration of hypotension before initiation of effective antimicrobial therapy is the critical determinant of survival in human septic shock. Crit Care Med 2006;34:1589-96.

13. Parkins MD, Sabuda DM, Elsayed S, Laupland KB. Adequacy of empirical antifungal therapy and effect on outcome among patients with invasive Candida species infections. J Antimicrob Chemother 2007;60:613-8.

14. Silvestri L, van Saene HK, de la Cal MA, et al. Surviving sepsis campaign needed consensus to exclude selective decontamination of the digestive tract. Crit Care Med 2008;36:2716-7.

15. Dellinger RP, Levy MM, Carlet JM, et al. Surviving sepsis campaign: International guidelines for management of severe sepsis and septic shock: 2008. Crit Care Med 2008;36:296-327.

16. Muscedere J, Dodek P, Keenan S, Fowler R, Cook D, Heyland D. Comprehensive evidence-based clinical practice guidelines for ventilator-associated pneumonia: prevention. J Crit Care 2008;23:126-37.

17. Bonten MJ. Selective digestive tract decontamination - will it prevent infection with multidrug-resistant gram-negative pathogens but still be applicable in institutions where methicillin-resistant Staphylococcus aureus and vancomycin-resistant enterococci are endemic? Clin Infect Dis 2006;43(Suppl 2):S70-4. 


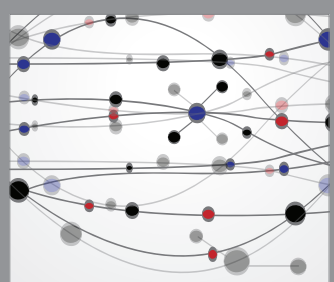

The Scientific World Journal
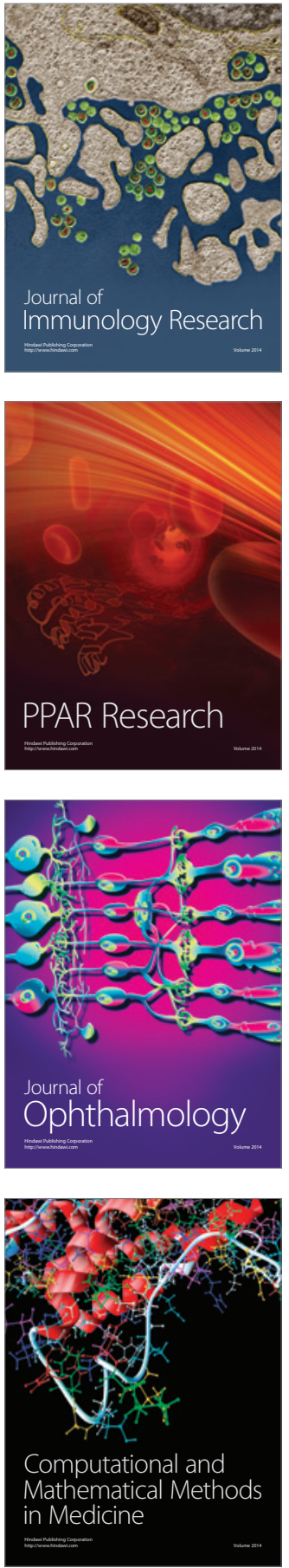

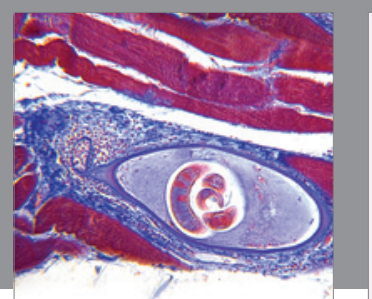

Gastroenterology Research and Practice

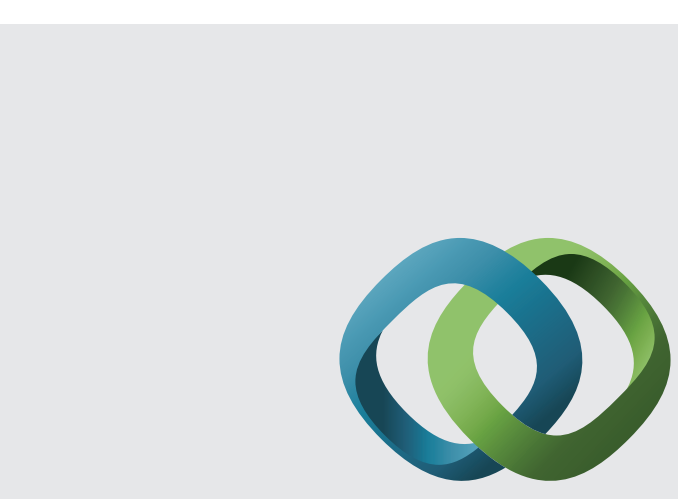

\section{Hindawi}

Submit your manuscripts at

http://www.hindawi.com
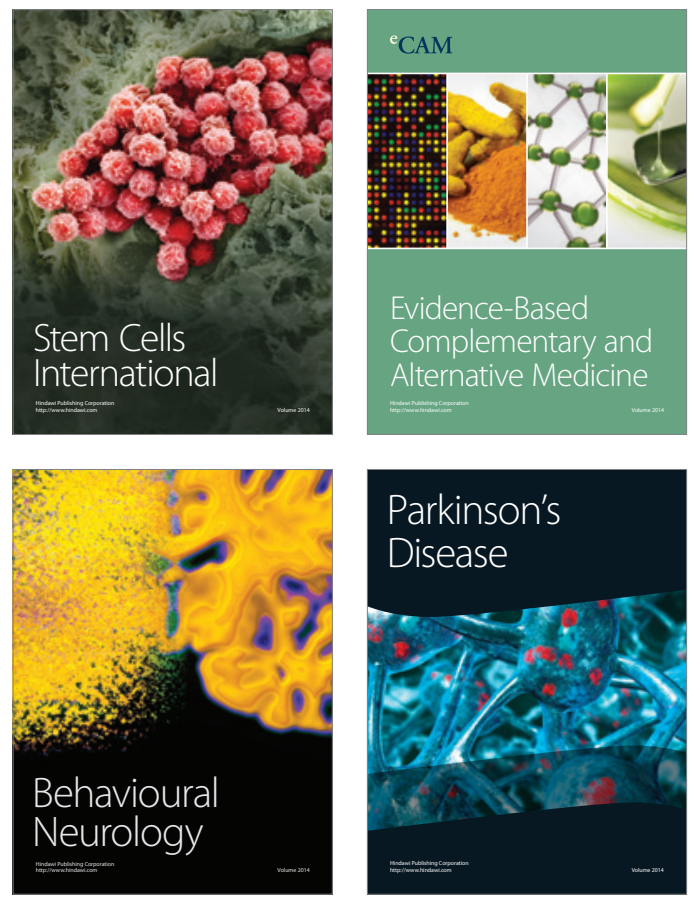
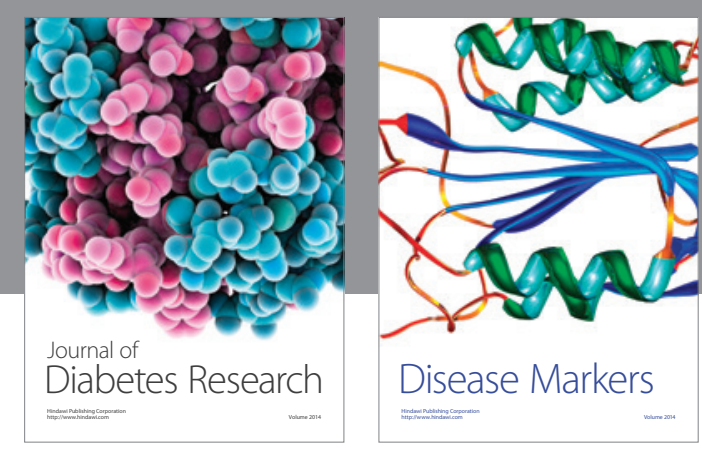

Disease Markers
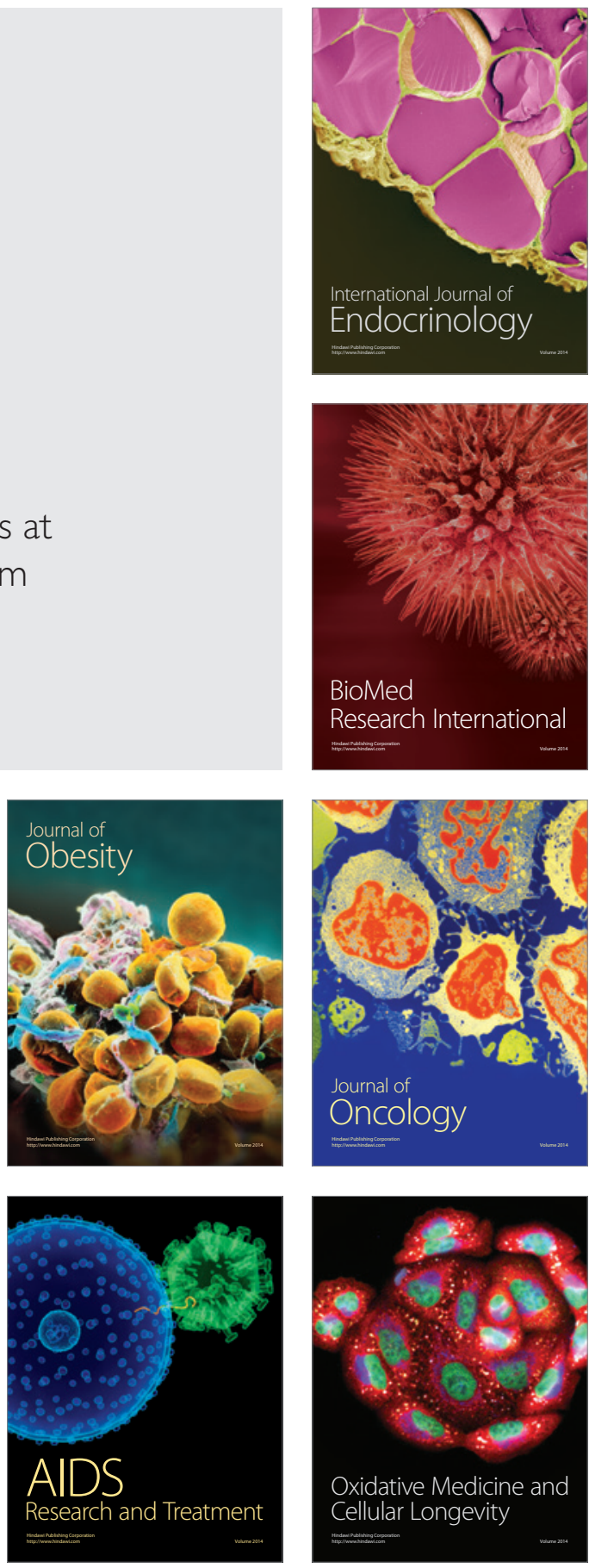